\title{
Prevención del riesgo de caída en adultos mayores con programa Kunte durante confinamiento por COVID-19 \\ Prevention older adults fall risk with Kunte program during COVID-19 confinement
}

\author{
*Manuel Villarroel Cerda, **Cristian Díaz Escobar, ***Matías Pozo Nuñez, ****Víctor Díaz-Narváez \\ *Centro de Salud Integral San José (Chile), **Asociación Nacional de Fútbol Profesional (Chile), ***U niversidad \\ Bernardo 0 Higgins (Chile), ****U niversidad Andres Bello (Chile)
}

\begin{abstract}
Resumen. Introducción: Lafaltade actividad físicae incremento de conductassedentarias duranteel confinamiento domiciliario de adultos mayores, debido al COVID-19, tendría como consecuencia, con el tiempo, un aumento del riesgo de caída en esta población. 0 bjetivo: Disminuir el riesgo de cáda en un grupo de adultos mayores con confinamiento domiciliario aplicando el programa de actividad física Kunte, concerniente en secuencias de movimientos de tipo oriental estructurados de acuerdo a fundamentos teórico-prácticos de laterapiafísica y metodológico-pedagógicos de la Educación Física. M étodos: Estudio de diseño exploratorio y cuasi experimental; aplicado en 35 adultos mayores (19 mujeres - 16 hombres), pertenecientes a la comuna de M aipú, Santiago, Chile. Durante un periodo de 3 meses, utilizando el T imed U p and Go y Prueba deT inetti antes y después de la intervención con el programa Kunte. Resultados: Los estadísticos descriptivos determinaron en un antes y después de aplicado el programa Kunte diferencias significativas $(p<0,05)$ y al tamente significativas $(p<0,005)$ parala prueba Tinetti y Timed Up and Go Test respectivamente, tanto en hombres como mujeres, en la valoración del riesgo de caída de adultos mayores; disminuyendo significativamente este riesgo en cuanto a su relación con el equilibrio corporal dinámico y la marcha. Conclusión: Laaplicación del programaKunte presentó resultadossignificativamente positivosal val orar el equilibrio corporal dinámico y la marcha, mediante pruebas funcionales relacionadas con el riesgo de caída en adultos mayores, en este caso con confinamiento domiciliario durante la pandemia por COVID-19.
\end{abstract}

Palabras claves: Covid-19, adulto mayor, riesgo de caída.

\begin{abstract}
Introduction: Lack of physical activity and increased sedentary behaviors during home confinement of older adults, due to COVID-19, would result, over time, in an increased risk of falling in this population. 0 bjective: To reduce the risk of falling in a group of older adults in home confinement by applying the Kunte physical activity program, based on sequences of oriental-type movements structured according to the theoretical-practical foundations of physical therapy and the methodological-pedagogical principles of Physical Education. Methods: Exploratory and quasi-experimental design study; applied in 35 older adults (19 women - 16 men), belonging to the commune of Maipú, Santiago, Chile. During aperiod of 3 months, using theT imed U $p$ and $G o$ andT inetti'sTest before and after the inter vention with the Kunte program. Results: Descriptive statistics determined significant $(p<0.05)$ and highly significant $(p<0.005)$ differences for theT inetti andT imed Up and Go Test respectively, in both men and women, in the assessment of the risk of falling of older adults; significantly decreasing this risk in terms of its relationship with dynamic body balance and walking Conclusion: The application of the Kunte program showed significantly positive results when assessing dynamic body balance and gait, through functional tests related to fall risk in older adults, in this case with home confinement during the COVID-19 pandemic.
\end{abstract}

Keywords: Covid-19, older adult, risk of fall.

\section{Introducción}

A fines del año 2019 las autoridadeschinasinformaron a la O rganización Mundial de la Salud (OMS) sobre un brote de neumonía en la ciudad deW uhan, denominado posteriormente enfermedad de COVID-19, generada por el virus SARS-CoV-2 capaz de provocar síndrome respiratorio agudo severo. Con alta capacidad de

Fecha recepción: 03-01-21. Fecha de aceptación: 16-03-21 Víctor Patricio Díaz Narváez vpdiaz@tie.cl transmisibilidad entre los seres humanos a través de las secreciones respiratorias de un sujeto infectado (sintomático, presintomático o asintomático) o por contacto con superficies contaminadas. Con el paso del tiempo lasituación terminó colapsando sistemas de sal ud, generando crisis sociales y económicas en el mundo, declarándose finalmente estado de pandemia (Cuestas \& M inassian, 2020; Listaet al. , 2020; M ojicaet al. , 2020; Serra, 2020).

La complejidad del COVID-19, según U rzúa et al. (2020), implica el virus en sí mismo, su rapidez de 
contagio, mayor riesgo para ciertos grupos y deficiencia en la respuesta sanitaria de urgencia frente a una alta demanda. Al no disponer se de una vacuna o tratamiento específico para este coronavirus, se ha establecido como estrategiade mayor eficiencialacuarentena, permitiendo con el confinamiento la protección de población vulnerable como puede ser el caso de losadultos mayores (AM) (Sánchez et al., 2020; Rodríguez et al., 2020; Antilao, 2020). Población que presenta alto riesgo en cuanto a consecuencias nocivas y mortalidad, porque pueden confluir en comorbilidades (hipertensión arterial, diabetes, cardiopatía coronaria, entre otras); síndromes geriátricos, grado de fragilidad, discapacidad y dependencia (Sepúlveda et al., 2020; Pinazo, 2020; Blanco \& Blanco, 2020; Tarazona et al ., 2020; Galaviz et al., 2020).

El confinamiento prolongado de la población y en especial para los AM ha tenido como resultado el incremento de conductas sedentarias y reducción de la actividad física; en paralelo a alteraciones emocionales como: peor estado emocional vinculado con sintomatología depresiva, trastornos del sueño, temor por la incertidumbre, entre otros (De la Cámara et al., 2020; Machado et al. , 2020; Woods et al. , 2020). Situación preocupante paraYamada et al. (2020), quienes reportan que, según la O MS, la participación social del AM mantiene las funciones físicas y mentales necesarias para una mejor autoval encia producto de la actividad física regular, adecuado soporte emocional, estimulación cognitiva e incremento de la autoestima. Este confinamiento por pandemia para los AM estaría generando en lo físico: una pérdida gradual de la masa y fuerzamuscular, alteración del balance corporal y función neuromuscular (Machado et al., 2020; Yamada et al., 2020). Lo que, en específico, de acuerdo a De La Cámara et al. (2020) incrementaría el riesgo de caída de estas personas durante y después de la cuarentena.

Las caídas son una amenaza significativa en la calidad de vida de los AM generando declive del autocuidado, disminución de la actividad física y aislamiento. Consideradas un síndrome geriátrico que puede provocar lesiones, discapacidad e incluso la muerte. Son de causalidad multifactorial como: edad avanzada, género femenino, vivir sólo, temor a caerse, comorbilidades, estado mental, alteración del balance corporal, pérdida de fuerza muscular, entre otras (Moles et al., 2020; Jo et al., 2020; Chen et al., 2020). No obstante, paraYe et al. (2020) pese a ser un problema de salud complejo, las caídas pueden ser prevenibles en losAM con programas de intervención que involucren actividad física, educación y seguridad del entorno. De acuerdo a Martínez et al. (2020), la OMS plantea que el envejecimiento activo, entendido como un proceso que optimiza las oportunidadesen salud, seguridad y participación mejora la calidad de vida del AM, realzando su potencial físico, mental y social; donde el ejercicio físico tendría una influencia positiva en las funciones físicas y psico-sociales de la persona. Especialmente cuando el ejercicio físico es practicado de manera regular a una intensidad adecuada y apropiadamente dirigido, resultando en un tratamiento no farmacológico efectivo y preventivo para varias patologías prevalentes en los AM (Martinez et al., 2020; M onteagudo et al., 2020). Además, los programas de ejercicios físicos aplicados correctamente se establecen como una estrategia económica, accesible y sin efectos adversos (M onteagudo et al., 2020).

En el caso de los programas de ejercicios para prevenir caídas en AM, según Chen et al. (2020), son variados y en general se orientan al control del balance corporal e incremento de la fuerza para miembros inferiores. Como parte de estas intervenciones, el Tai Chi se destaca por ser un sistema de ejercicios tradicionales chinos que integra el trabajo corporal y mental, de intensidad baja a moderada, centrado en movimientos coordinados suaves de bajo impacto; con suficiente evidencia científica para ser recomendados en AM al provocar mejoras en el balance corporal, la fuerza muscular, propiocepción y adaptaciones posturales; además de influir en el aspecto mental reduciendo el estrés, ansiedad, alteraciones del ánimo, depresión y aumentar la autoestima (Chen et al., 2020; Shi et al., 2020; Wang et al., 2020). Con características similares a los movimientos que contiene el Tai Chi, en Chile se elaboró el programa denominado Kunte. En el cual se integró técnicas de manos y posiciones desarrolladas desde un estilo de arte marcial oriental conocido como Kung Fu, en conjunto con elementos de la terapia física y prevención en salud de la kinesiología, además de principios metodológicos-pedagógicos del entrenamiento físico desde la Educación Física; aplicándose como taller comunitario en un grupo de AM que participaba en una sede social comunal en la ciudad de Santiago, Chile; evidenciándose mejora en su independencia funcional, capacidad cognitiva y disminución del riesgo de caída (Díaz et al., 2017). El propósito del estudio, fue aplicar nuevamente el Kunte como programa preventivo para riesgo de caídas en un grupo de AM confinados en sus hogares, debido a la cuarentena establecida en el país, durante la pandemia por COVID-19. 


\section{Material y método}

Estudio de diseño exploratorio y cuasi experimental (Díaz \& Calzadilla, 2016), aplicado por tres meses durante el segundo semestre año 2020, cuando las medidas sanitarias por COVID-19 per mitieron un mayor control de los contagios en la ciudad de Santiago, Chile. La muestra final (no aleatoria y por conveniencia) se constituyó de 35 sujetos (19 mujeres, media en edad: $67,9+2,6$ años - 16 hombres, media en edad: 67,4 + 2,9 años) con residencia en la Villa Juan Cerda y participación en el club social Carmelitas, comuna de Maipú.

Inicial mente, por víatelefónica, se contactó a 43 AM que participaban activamente en la sede social; 35 de ellos accedieron al estudio, quienes posteriormente firmaron el consentimiento informado correspondiente, además de ser informados de la posibilidad de retiro voluntario, durante el proceso, sin ningún tipo de compromiso. El estudio fue aprobado por el Comité de Ética de la U niver sidad Europea del Atlántico (España), consignado en el actaCE-22 correspondiente al a sesión del 19 de Junio de 2020. De las ocho personas que se excluyeron del estudio, cinco se excusaron por temor al contagio y cuatro no especificaron el motivo.

Durante el contacto telefónico se consultó por una aplicación del programa Kunte vía online o presencial; determinándose presencial por la falta de manejo tecnológico del grupo. Los investigadores, ambos kinesiólogos certificados en el programa Kunte nivel básico para AM, ejecutaron los procedimientos domiciliarios cumpliendo con los permisos correspondientes y medidas de protección establecidas por laautoridad sanitariadel paísen cuanto a: trajeclínico, mascarilla N K 95 desechable, pechera desechable, guantes de procedimiento desechables, alcohol gel al $95 \%$ de alcohol etílico, desinfección con amonio cuaternario para calzado, ropa u objetos que se movilizaran en los domicilios para realizar los procedimientos y en lo posible manteniendo una distancia mínima de dos metros con las personas.

Las instrucciones de los procedimientos, asociado a los ejercicios específicos del Programa Kunte, dado a los AM de la muestra fue realizado por un solo Kinesiólogo (especializado en Gerontología) debidamente entrenado previamente en este tipo de programa. Las mediciones antes y después de aplicado el programa antes señalado fue realizado por otro Kinesiólogo (especializado en Gerontología) y también debidamente entrenado en las técnicas de medición. La especificidad en la participación de losKinesiólogosantes descritos fue realizada con el objeto de evitar sesgo en la medición.

Los procedimientos se realizaron en días hábiles de lunes a viernes, previa coordinación entre los investigadores y el grupo, de acuerdo al a disponibilidad semanal para realizar las dos sesiones de 30 a 40 minutos respectivas; según número de AM y condiciones del espacio físico por domicilio. Las dos primeras y últimas dos semanas del proceso se destinaron para registro de datos y evaluación del riesgo de caída; procedimiento que fue ejecutado siempre por el mismo investigador y el otro investigador aplicó el programa Kunte durante las ocho semanas intermedias.

En la evaluación del riesgo de caída se aplicó: a) Timed Up and Go Test: sujeto sentado en una silla con respaldo y sin apoya brazos, se incorporó y caminó en línea recta hasta rodear un elemento marcador a 3 metrosdedistancia, volviendo al punto y posición inicial. El desplazamiento debió ser lo más rápido posible, registrándoseel tiempo en segundos. La relación tiempo y riesgo de caída se estableció en: 10 o menos segundos como valores normales, es decir, mínimo riesgo de cáda, entre 11 a 20 segundos riesgo moderado y sobre 20 segundos riesgo alto (M ancillaet al., 2015). b) Escala de Tinetti: evaluación que consigna valoración del equilibrio (estático-dinámico) y la marcha mediante observación y consultas. La escala presenta puntuación máximade 12 paraítem de marchay 16 para equilibrio, con una suma total de 28 puntos. Consignándose riesgo alto de cá́da con menos de 19 puntos; riesgo moderado entre 19 a 23 puntos y mínimo riesgo con 24 o más puntos (Díaz et al., 2017; Carballo et al., 2018).

El programa Kunte para domicilio se estructuró principalmente en dos fases y según progresión individual de los adultosmayores. Seinició con ejercicios para miembros superiores con la persona sentada en una silla realizando movimientos en el plano sagital, frontal y horizontal, de manera simétrica y asimétrica sin elevar los segmentos por sobre la altura de los hombros; alternando con ejercicios para miembros inferiores desde posición bípeda ejecutando desplazamientos mediante posiciones básicas de Kunte, dentro de una base de sustentación estable, principalmente en el plano frontal y sagital; agregando progresivamente ejercicios de estabilidad con o sin uso de asistenciaexterna, por ejemplo lamismasillautilizada en lasesión. Lassecuencias de ejercicios paracada sesión, alternaban ejercicios ya trabajados en la sesión anterior con nuevos movimientos, variando también el volumen 
de trabajo en cuanto a número de repeticiones, series y tiempo de descanso según condición particular de cada AM. Tanto los movimientos de miembros superiores como inferiores entrenados en primera instancia, de maneraindependiente, se fueron uniendo y combinando a medida que avanzaban las sesiones generando secuencias simples de movimientos ordenadas por números (secuencia 1, secuencia 2, consecutivamente). En el caso de los ejercicios de estabilidad continuaron con el uso de un elemento externo, generalmente una silla, aumentándose el volumen de trabajo en tiempo durante las posiciones unipodales. En la segunda fase (cuatro semanas antes de la evaluación post intervención) se inició la combinación de los ejercicios entrenados en la primera fase; uniendo los movimientos de miembros superiores practicados en posición sedente o bípedo con las posiciones básicas de Kunte, para terminar concretando ejercicios denominados en el programa como Formas Kunte donde se desarrollan de manera coordinada y fluida movimientos de miembros superiores e inferiores al mismo tiempo mientras se ejecuta un diseño de piso simple formando una figura en cruzo en cuadrado sobre el piso, tanto al lado derecho como izquierdo o hacia adelante 0 atrás. Ejercicios que en su conjunto se aplicaban para trabajar el ámbito físico y a su vez involucrar el aspecto mental de la persona, solicitándole unir secuencias de movimiento practicadas en las sesiones anteriores y en al gunos casos, según condición individual y como actividad complementaria, proponer la elaboración de una secuencia simple utilizando movimientos enseñados en el programa por parte de la misma persona; a fin de estimular la motivación y concentración de la persona durante la sesión (Díaz et al., 2017).

\section{Análisis estadístico}

Los datos primarios fueron sometidos a estudios de normalidad (Prueba de Shapiro-Wilk: S-W) y de homocedasticidad (Levene: L). La comparación entre los datos obser vados antes y después de la intervención, en concordancia con la edad, fueron comparados mediante la prueba de rangos con signo de W ilcoxon para dos muestras relacionadas.

Se estimaron las medias, desviación estándar (DS), valores mínimos y máximos en cada prueba, el estadístico Z y la probabilidad de cometer el Error de Tipo I en una prueba bilateral y unilateral. El nivel de significación empleado fue de ád»0.05 para pruebas exactas bilaterales y unilaterales.

\section{Resultados}

En la entrevista inicial, de los 35 AM que constituyeron el grupo de intervención: el 30\% vivía sólo y del $70 \%$ restante, el $62 \%$ correspondía a parejas deAM que vivían solos. El 83\% del grupo indicó evitar salir por miedo al contagio, tener temor a las caídas domiciliarias y posible incapacidad de incorporarse por sí mismo. El 58\% declaró haber presentado caídas previo al estudio con consecuencias físicas de distinta gravedad y en general, indicaron haber disminuido el nivel de actividad física durante el confinamiento.

Los resultados de las pruebas de S-W y L fueron significativos $(p<0.005)$. Como consecuencia, los datos no se distribuyen normal mentey no son homocedásticos y justifica y explica la prueba estadística empleada para las comparaciones en el presente trabajo.

Los resultados de la estimación de los estadísticos descriptivos, así como de lacomparación antesy después de la prueba de Tinetti y de Timed Up and Go en hombres se presentan en laTabla 1. En ambos casos se determinaron diferencias significativas $(p<0.05)$ y altamente significativas $(p<0.005)$ en las pruebas exactas bilaterales y unilaterales, todo lo cual confirma que las medias de las pruebas antes y después son estadísticamente diferentes, especialmente importante

\footnotetext{
Tabla 1.

Resultados de la comparación de la prueba de Tinetti y Timed Up and Go, antes y después de la intervención, con programa Kunte en AM hombres.

Hombres $n$ Media DS Valor Valor Estadistico Bilateral Unilateral \begin{tabular}{cccccccrc} 
& & & & Mínimo & Máximo & Z & exacta & exacta \\
\hline Tinetti Pre & 16 & 18.81 & 2.64 & 15 & 24 & $-3,53$ & 0.0005 & 0.0005
\end{tabular} $\begin{array}{llllll}\text { Tinetti Pre } & 16 & 18.81 & 2.64 & 15 & 22 \\ \text { Tinetti Post } & 16 & 22.63 & 2.94 & 18 & 27\end{array}$ $\begin{array}{cllllllll}\text { Tinetti Post } & 16 & 22.63 & 2.94 & 18 & 27 & & & \\ \text { TUGPre } & 16 & 18.69 & 3.94 & 12 & 25 & -2,39 & 0.014 & 0.007\end{array}$ $\begin{array}{llllll}\text { TUG Post } & 16 & 15.69 & 4.30 & 8 & 20\end{array}$ TUG:Timed UP and Go; AM: Adultos mayores

Tabla 2 .

Resultados de la comparación de la prueba de Tinetti y Timed U p and Go antes y después de la intervención, según grado de riesgo de caída en AM hombres

Hombres n Media DS Valor Valor Estadistico Bilateral Unilateral \begin{tabular}{ccccccccc} 
& & & & Mínimo & Máximo & Z & Exacta & Exacta \\
\hline Tinetti Pre & 16 & 1.62 & 0.62 & 1 & 3 & -3.05 & 0.002 & 0.001
\end{tabular} $\begin{array}{cllllllll}\text { Tinetti Post } & 16 & 2.31 & 0.60 & 1 & 3 & & & \\ \text { TUG Pre } & 16 & 1.62 & 0.50 & 1 & 2 & -3.16 & 0.002 & 0.001\end{array}$ $\begin{array}{lllllllll}\text { TUG Pre } & 16 & 1.62 & 0.50 & 1 & 2 & -3.16 & 0.002 & 0.001 \\ \text { TUG Post } & 16 & 2.25 & 0.48 & 2 & 3 & & & \end{array}$ TUG:Timed Up and Go; Valor 1: Alto riesgo de caída; Valor 2: Moderado riesgo de cáída; Valor 3: Bajo riesgo de caída. AM: Adultos mayores

Tabla 3.

Resultados de la comparación de la prueba de T inetti y Timed U p and Go antes y después de la intervención, con programa Kunte en AM mujeres

Mujeres $n$ Media DS Valor Valor Estadistico Bilateral Unilateral \begin{tabular}{ccccccccc} 
& & & & Mínimo & Máximo & Z & Exacta & Exacta \\
\hline Tinetti Pre & 19 & 19.0 & 3.46 & 14 & 25 & -3.62 & 0.0001 & 0.0001
\end{tabular} $\begin{array}{llllll}\text { Tinetti Post } & 19 & 23.74 & 2.51 & 20 & 29\end{array}$ $\begin{array}{llllllll}\text { Tinetti Post } & 19 & 23.74 & 2.51 & 20 & 29 & & \\ \text { TUG Pre } & 19 & 18.11 & 5.46 & 8 & 25 & -2.51 & 0.012\end{array}$ TUG Post 19 14.47 $4.23 \quad 8 \quad 20$ TUG:Timed Up and Go; AM: adultos mayores

Tabla 4

Resultados de la comparación de la prueba de Tinetti y Timed Up and Go antes y después de la intervención, según grado de riesgo de cá́da en AM mujeres

Mujeres $n$ Media DS Valor Valor Estadístico Bilateral Unilateral \begin{tabular}{ccccccccc} 
& & & & Mínimo & Máximo & $Z$ & exacta & exacta \\
\hline Tinetti Pre & 19 & 1.68 & 0.75 & 1 & 3 & -3.36 & 0.001 & 0.0005
\end{tabular} $\begin{array}{llllll}\text { Tinetti Post } & 19 & 2.53 & 0.51 & 2\end{array}$ $\begin{array}{lllllllll}\text { TUG Pre } & 19 & 1.68 & 0.67 & 1 & 3 & -2.97 & 0.003 & 0.002\end{array}$ $\begin{array}{llllll}\text { TUG Post } & 19 & 2.32 & 0.48 & 2 & 3\end{array}$

TUG:T imed Up and Go; Valor 1: Alto riesgo de caída; Valor 2: Moderado riesgo de cáda; Valor 3: Bajo riesgo de caída; AM: adultos mayores
} 
son los resultados de las pruebas exactas unilaterales que demuestran que una media es mayor que otra. En la Tabla 2 se muestran los resultados de las mismas pruebas, pero desde la perspectiva de la estimación del riesgo de caída en los hombres. Estos resultados son análogos a los descritos anteriormente. En resumen, las medias después de la intervención realizada son mayores que la media estimada antes de la inter vención (lo cual explica el signo menos del estadístico Z) en todas las comparaciones realizadas en los hombres.

En laTabla 3 y 4 se presentan los mismos resultados antes descritos, pero referidos específicamente a las mujeres. Estos resultados muestran que las medias de las pruebas observadas después de la intervención con Kunte son mayores que los valores observados antes del proceso, con diferencias significativas y altamente significativas ( $p<0.005$ y $p<0.0005$ respectivamente) y empíricamente estos resultados son similares al establecido en los hombres.

\section{Discusión}

La pandemia del COVID-19 ha generado una crisis mundial en los distintos ámbitos que conforman el desarrollo de las sociedades; especialmente en los sistemas de salud de muchos países. De las estrategias establecidas para controlar la proliferación del virus en la población, con especial cuidado para los grupos de mayor riesgo, donde se integra a losAM, la cuarentena o confinamiento domiciliario se ha consignado como la acción más efectiva mientras no exista una solución definitiva (Cuestas \& M inassian, 2020). Sin embargo, para Machado et al. (2020), el encierro obligatorio cercano a los tres meses conlleva un aumento en el declive de las funciones físicas y psicológicas de losAM; situación que debiese ser considerada como un problema de prioridad sanitaria, especialmenteen los países donde la cuarentena ha superado este periodo de restricción. Esta determinación sanitaria también involucró la suspensión de los programas de actividad física comunitarios aplicados en AM; por lo que Said et al. (2020), recomendaron canalizar los esfuerzos en intervenciones de tipo domiciliario, con objetivos orientados al entrenamiento de las capacidades físicas, manteniendo o mejorando las habilidades funcionales de los AM, a fin de mitigar la pérdida de movilidad y autonomía. Planteamiento respal dado por Antilao (2020) y Yamada et al. (2020), quienes manifestaron la importancia en el diseño e implementación de estrategias que incentiven este tipo de intervención, como factor clave para superar el COVID-19 y las secuelas que dejará esta crisis sanitaria. Entendiéndose que la etapa de la vejez, según Tejada et al. (2020), debe ser una prioridad en salud pública parala mantención de la calidad de vida y aumento de los años de autonomía en esta población.

Antes de la pandemia, el tema de la vejez tenía prioridad en muchos países y lo seguirá teniendo. No obstante, en la actualidad los requerimientos sanitarios están enfocados en problemáticas de mayor urgencia como: el colapso de los centros clínicos, el agotamiento del personal de salud, campañas de vacunación, manejo de las crisis económicas y sociales que el COVID-19 ha provocado. Por lo que considerar la posibilidad de destinar recursos humanos y materiales para programas de actividad física, de tipo comunitario como domiciliario, en la prevención del declive funcional de IosAM, resulta en estos momentos alo menoscomplejo. Lo que no imposibilita, dirigir esfuerzos en programar o diseñar futuras estrategias de intervención, a corto, mediano y largo plazo, enfocadas en esta materia para ser aplicadas cuando las estrategias sanitarias lo permitan.

La actividad física, en sus distintas expresiones, siempre será beneficiosa para el ser humano, a lo largo de su ciclo de vida. Pese a lo positivo que resulta el ejercicio físico regular, Dhami et al. (2015), expusieron que el retiro de los AM que iniciaban programas de actividad física era superior al 50\% antes de cumplirse los seismeses de intervención, indicándose como factores de retiro: la falta de apoyo social, el estado de salud, grado de motivación y percepción de entretenimiento por parte de la persona. Aunque el tiempo de aplicación fue menor a un semestre en el estudio, todos los AM completaron el proceso de intervención, expresando en su mayoría el interés de retomar el Kunte una vez superada la crisis sanitaria. Exponiéndose este resultado como satisfactorio para el proyecto, por las condiciones en que se debió desarrollar debido a la cuarentena, asumiendo posibles problemáticas en los AM a nivel físico, emocional, económico y social durante este periodo y la logística requerida para ejecutar cada sesión domiciliaria en relación a permisos, coordinación de horarios, cumplimiento de normas sanitarias, entre otros. Incluso, sobrellevando lo planteado por De la Cámara et al. (2020), quienes indicaron que otro factor relacionado con el incremento de la inactividad física, al iniciarse la pandemia, fue generado por el temor de contagio producto de la interacción, especialmente por contacto, con otras personas o posibles superficies contaminadas durante la ejecución de los programas de 
ejercicios. Situación que fundamentó la decisión expresadapor laspersonasqueseexcusaron de participar en el estudio. No obstante, pese al miedo o incertidumbre generada por el COVID-19 en la población y en especial en los AM, la intervención realizada con todas las medidas de seguridad y adecuado manejo por parte de los investigadores permitió que el proceso terminara positivamente para la totalidad de los participantes.

El estudio determinó que la batería de ejercicios establecidos en el programa Kunte, con el objetivo específico de prevenir caílas en un grupo de AM, con confinamiento domiciliario durante la cuarentena, presentaran unareducción significativadel riesgo de caída de las personas, tanto en mujeres como hombres; evaluado mediante el Timed U $p$ and Go Test $(p<0.005)$ y PruebadeT inetti $(p<0.05)$ midiendo un antesy después de aplicar el Kunte. Demostrándose una mejoría del equilibrio corporal dinámico en la primera prueba y de la marcha además del equilibrio corporal en la segunda. Antecedente que se destaca en el caso de las mujeres, en el entendido que según género las mujeres tienen mayor riesgo de cáda (Moles et al., 2020; Jo et al., 2020). Resultados que tuvieron como eje central ejercicios con desplazamientos corporales simples en distintas direcciones, trabajos de equilibrio unipodal con asistenciaexterna, movilidad y amplitud de movimientos para miembros superiores, dentro de un marco de acciones motoras de influencia oriental que se fueron encadenando progresivamente mediante secuencias de movimientos coordinados denominados Formas Kunte. Ejercicios que a su vez se adecuaron a las capacidades físicas individuales y estados emocionales de los AM durante cada sesión, procurando que las personas no se adaptaran al Kunte, por el contrario, que el programa Kunte continuamente se fuera ajustando a cada participante. Además, se consigna que la condición singular en quese aplicó el estudio, respal dalosresultados obtenidos en cuanto a que los AM intervenidos llevaban un confinamiento total en sus hogares por lo menos de dos meses, lo que significó restricción total de las actividades que realizaban rutinariamente, disminuyendo la actividad física que realizaba habitualmente e incrementando actitudes sedentarias en el hogar (De la Cámara et al., 2020; Machado et al., 2020; Woods et al., 2020); por lo que las dos sesiones de Kunte semanales se constituyeron, para la mayoría de losAM, en la única actividad física controlada y dirigida que realizaban. Por otra parte, esta metodología fue también aplicada en una intervención con Kunte en modalidad de taller comunitario para un grupo deAM, presentándose al final del proceso una disminución del riesgo de cáida; el grupo también declaró sentirse más autónomos después delaintervención, con mejor estado de ánimo y con el expreso interés de continuar participando en esta modalidad de intervención (Díaz et al., 2017). Lo que sustenta, de acuerdo aAraque et al. (2020), que los programas multicomponentes que integran ejercicios físicos combinados de fuerza, equilibrio, flexibilidad, coordinación, entre otros; mejoran la agilidad y el balance corporal relacionados con la disminución del riesgo de caída, junto al aumento de la autoestima y bienestar emocional durante la ejecución de actividades de la vida diaria, promoviendo la calidad de vida de los AM. Efectos que si bien son factibles de lograrse con diferentes modalidades de ejercicios físicos; en el caso del programa Kunte se ha demostrado beneficios similares a los presentados por intervenciones con influencia oriental como el Tai Chi, mejorando el balance corporal y disminuyendo el riesgo de caída (Wang et al. , 2020). Por lo que, en consecuencia a lo planteado por Shi et al. (2020), el Tai Chí aśí como esta propuesta denominada Kunte, podría ser recomendada a futuro como una alternativa efectiva y segura paralarecuperación física post COVID-19, como lo serían para Shi et al. (2020) las intervención con ejercicios de Tai Chi; reforzado según lo expuesto por Chen et al. (2020) quienes describen el envejecimiento como un proceso individual que amerita de una prescripción de ejercicios en función al estado de salud y funcionalidad de cada persona, centrado en una medición objetiva y adecuada supervisión para incrementar la complejidad eintensidad de los estímulos de manera gradual. Añadiéndose además, que el programa Kunte, al igual que otras alternativas de influencia oriental, se ha descrito como un programa de ejercicios físicos de bajo costo en cuanto a recursos materiales y humanos (Díaz et al. 2017).

El envejecimiento saludable según Tornero et al. (2020) no sólo seríarelativo ala salud física, comprende una motivación por parte de la persona para lograr sentirse satisfecho con su vida en general, con las actividades motoras que realiza, con las relaciones familiares y con su entorno; lo que se compromete cuando existe predominio del sedentarismo y la inactividad física. La prioridad durante la pandemia por COVID-19 amerita continuar evitando los contagios hasta tener una solución definitiva como una vacuna, principalmente para las personas de riesgo como son Ios AM. Sin embargo, el declive funcional y por 
consiguiente el incremento del riesgo de caída en esta población seguirásiendo un problemarelevanteen salud pública, que debe ser considerado durante y después de lograr superar esta crisis sanitaria. El programa Kunte, si bien ha presentado resultados positivos aplicándose primero como un taller comunitario y en el presente estudio como taller domiciliario, todavía debe seguir siendo sometido a nuevos proyectos de investigación para consolidar esta iniciativa como una adecuada alternativa en estrategias de prevención en salud geriátrica relacionadas con la actividad física saludable.

Como limitaciones del estudio, una de estas se asociaría al error de muestreo que se presenta en todos los estudios con muestras relativamente pequeñas, como acontece en este caso. Sin embargo, las diferencias encontradas entre las medias comparadas, exponen que existen tendencias bien fundamentadas que pueden estar relacionadas con una acción positiva de la intervención respecto a las variables estudiadas. 0 tra limitación se relaciona con la complejidad existente dada por la crisis del COVID-19 en cuanto a la posibilidad de estudiar otras variables o registrar más antecedentes de la muestrao del proceso durante el periodo de aplicación, debido a que la prioridad fue velar por la salud y seguridad por parte de los investigadores hacia el grupo de AM, especialmente evitando el contacto e incomodidad de ellos tanto en las evaluaciones como en las sesiones de Kunte.

Por lo tanto, las alternativas futuras de intervención debiesen tener consignado dos propósitos principales referidos a estudiar una muestra mayor que permita confirmar las marcadas tendencias establecidas en el presente trabajo y en un periodo de aplicación, con un mínimo de 6 meses, relacionado con el grado de adherencia de las personas hacia esta propuesta. Sumado a otras variables de investigación, factibles de considerar, sin las limitaciones y exigencias sanitarias impuestas durante el manejo de la pandemia por COVID-19.

\section{Conclusión}

En la prevención de riesgo de caídas de un grupo de adultos mayores confinados en sus domicilios, debido a la pandemia por COVID-19, el programa Kunte presentó resultadossignificativamente positivosal evaluar el equilibrio corporal dinámico y la marcha mediante la aplicación de dos pruebas funcionales, antes y después de la intervención con Kunte. Sin embargo, se requiere de nuevas intervenciones que continuen consolidando el programa como una alternativa viable y segura en la prevención de caídas para esta población, durante y después de superada la crisis sanitaria.

\section{Referencias}

Antilao P., Luis. (2020). Actividad física y brote de Coronavirus ¿Q ué medidas se adoptarán para el adulto mayor en Chile?. Revista Médica de Chile, 148(2), 271-272. https:// dx. doi.org/ 10.4067/ s0034-98872020000200271

Araque-Martínez, M., Ruiz-M ontero, P., \& Artés-Rodríguez, E. (2020). Efectos de un programa de ejercicio físico multicomponente sobre la condición física, la autoestima, la ansiedad y ladepresión depersonas adultas-mayores. Retos, 39, 1024-1028. https: / / doi. org/ 10.47197/ retos. v0i39.83282

Blanco-Tarrio, E., \& Blanco Sánchez, G. (2020). Atención primaria y residencias de ancianos: a propósito de la COVID19. Semergen, 46 Suppl 1, 26-34. https:/ / doi.org/ 10.1016/ j.semerg. 2020.06.003

Carballo-Rodríguez, Adelina, Gómez-Salgado, Juan, CasadoVerdejo, Inés, O rdás, Beatriz, \& Fernández, Daniel. (2018). Estudio de prevalencia y perfil de caídas en ancianos institucionalizados. Gerokomos, 29(3), 110-116. Recuperado en 24 de diciembre de 2020, de http:/ / scielo.isciii.es/ sciel 0.php?script $=$ sci_arttext $\&$ pid $=\$ 1134$ 928X2018000300110\&lng=es $\delta$ tlng=es.

Chen, P. J., Penn, I. W., Wei, S. H., Chuang, L. R., \& Sung, W. H. (2020). Augmented reality-assisted training with selectedTaiChi movementsimproves bal ance control and increases lower limb musclestrength in older adults: A prospective randomized trial. Journal of Exercise Science and Fitness, 18(3), 142- 147. https:/ / doi.org/ 10.1016/ j.jesf.2020.05.003

Cuestas, M. L., \& M inassian, M. L. (2020). COVID-19: Ecos de una pandemia. Revista Argentina de M icrobiología, 52(3), 167168. https:/ / doi.org/ 10.1016/ j.ram.2020.09.003

De LaCámara, M. Á., Jiménez-Fuente, A., \& Pardos, A. I. (2020). Fallsin older adults: Thenew pandemic in the post COVID-19 era?. Medical Hypotheses, 145, 110321. https:/ / doi. org/ 10.1016/ j.mehy.2020.110321

Dhami, P., M oreno, S., \& DeSouza, J. F. (2015). New framework for rehabilitation - fusion of cognitive and physical rehabilitation: the hope for dancing. Frontiersin Psychology, 5, 1478. https:/ / doi. org/ 10.3389/ fpsyg.2014.01478

Díaz, C., Aceituno, C., Tudesca, N., Villarroel, M., Yánez, S., \& Llach, L. (2017). Estudio preliminar: programaKunte parala mantención y/ o mejoradelaindependenciafuncional deadultos mayores autovalentes. U BO H ealth Journal, 4(1): 41-52. https:/ / www.ubo.cl/ wp-content/ uploads/ 14518 revista_hearlth_journa_C.pdf

Díaz-Narváez, V. P., \& Calzadilla Núñez, A. (2016). Artículos científicos, tipos de investigación y productividad científicaen las Ciencias de la Salud. Revista Ciencias D e La Salud, 14 (01), 115-121. https:/ / doi. org/ 10.12804/ revsalud14.01.2016.10

Galaviz Berelleza, R., TrejoTrejo, M., Borbón Román, J., Alarcón Meza, E., Pineda Espejel, H., Arrayales Millan, E., Robles Hernández, G., \& Cutti Riveros, L. (2020). Efecto de un programade entrenamiento de fuerzasobre IGF- 1 en adultos mayores con obesidad e hipertensión controlada. Retos, 39, 253-256. https: / / doi. org/ 10.47197/ retos. v0i39.74723

Jo, K. H., Park, J., \& Ryu, S.Y. (2020). The effects of mental health 
on recurrent falls among elderly adults, based on Korean Community Health Survey data. Epidemi ology and $\mathrm{H}$ ealth, 42, e2020005. https:/ / doi.org/ 10.4178/ epih. e2020005

Lista-Paz, A., González-D oniz, L., \& Souto-Camba, S. (2020). ¿Q ué papel desempeña la Fisioterapia en la pandemia mundial por COVID-19?. Fisioterapia, 42 (4), 167-169. https: / / doi. org/ $10.1016 /$ j.ft.2020.04.002

Machado, C., Pinto, R. S., Brusco, C. M., Cadore, E. L., \& Radaelli, R. (2020). COVID-19 pandemic is an urgent time for older people to practice resistance exercise at home. Experimental Gerontology, 141, 111101. https:// doi.org/ 10.1016/ j. exger. 2020.111101

Mancilla S, Eladio, Valenzuela H, José, \& Escobar C, Máximo. (2015). Rendimiento en las pruebas «Timed U p and Go» y «Estación U nipodal» en adultos mayores chilenos entre 60 y 89 años. Revista Médica de Chile, 143(1), 39-46. https: / / dx. doi. org/ 10.4067/ S0034-98872015000100005

MartínezH eredia, N. , SantaellaRodríguez, E. \& \& Rodríguez-García, A.-M. (2020). Beneficios delaactividad física parala promoción de un envejecimiento activo en personas mayores. Revisión bibliográfica. Retos, 39, 829-834. https:/ / doi.org/ 10.47197/ retos. v0i39.74537

M ojica-Crespo, R ., \& M orales-Crespo, M. M. (2020). Pandemia COVID-19, la nueva emergencia sanitaria de preocupación internacional: una revisión. Semergen, 46 Suppl 1, 65-77. https: / / doi. org/ 10.1016/ j. semerg. 2020.05.010

M olés Julio, M. P., Lavedán Santamaría, A., Botigué Satorra, T., Masot Ariño, O., Esteve Clavero, A., \& Maciá Soler, M. L. (2020). Characteristics and Circumstances of Falls in the Community-Dwelling Older Adult Population. Journal of Primary Care \& Community Health, 11, 2150132720940508. https: / / doi.org/ 10.1177/ 2150132720940508

M onteagudo Chiner, P., Herreros, J., Beltrán, J., Cordellat M arzal, A., Roldán Aliaga, A., \& Blasco-Lafarga, C. (2020). Retos y análisis de un programamunicipal de prescripción deejercicio físico para la salud: El programaVIU-ACTIU. Retos, 39, 993997. https:/ / doi.org/ 10.47197/ retos. v0i39. 77880

Pinazo-HernandisS. (2020). Impacto psicosocial de laCOVID-19 en las personas mayores: problemas y retos. Revista Española de Geriatría y Gerontología, 55(5), 249-252. https:/ / doi. org/ 10.1016/ j.regg.2020.05.006

Rodríguez, M. Á., Crespo, I., \& O Imedillas, H. (2020). Ejercitarse en tiempos de la COVID-19: ¿qué recomiendan hacer los expertos entre cuatro paredes?. Revista Española de Cardiología, 73(7), 527-529. https:/ / doi.org/ 10.1016/ j. recesp. 2020.04.002

Said, C. M. , Batchelor, F., \& Duque, G. (2020). Physical Activity and Exercise for Older People During and After the Coronavirus Disease 2019 Pandemic: A Path to Recovery. Journal of the American Medical Directors Association, 21 (7), 977-979. https:/ / doi.org/ 10.1016/ j.jamda.2020.06.001

Sánchez-Duque, J., Arce-Villalobos, L., \& Rodríguez-M orales, A. (2020). Enfermedad por coronavirus 2019 (COVID-19) en América Latina: papel delaatención primariaen lapreparación y respuesta. Aten Primaria. , 52(6):369-372. https:/ / doi. org/ 10.1016/ j. aprim. 2020.04.001

Sepúlveda-Loyola, W., Rodríguez-Sánchez, I., Pérez-Rodríguez, P., Ganz, F. , Torralba, R., O liveira, D. V., \& Rodríguez-M añas,
L. (2020). Impact of Social Isolation Due to COVID-19 on Health in Older People: Mental and Physical Effects and Recommendations. The Journal of N utrition, Health \& Aging, 110. Advance online publication. https:/ / doi.org/ 10.1007/ s12603-020-1469-2

SerraValdés, M A. (2020). COVID-19. Delapatogeniaalaelevada mortalidad en el adulto mayor y con comorbilidades. Rev H aban Cienc M éd [Internet], 19(3):e3379.

Shi,Y., Wen, D., Wang, H. , Zhang, P., Zhong,Y., Liu, D., \& Zhou, D. (2020).Tai Chi for coronavirus disease 2019 in recovery period: A protocol for systematic review and meta analysis. M edicine, 99(32), e21459. https:/ / doi. org/ 10.1097/ MD.0000000000021459

Tarazona-Santabalbina, F. J., M artínez-Velilla, N. , Vidán, M.T., \& García-Navarro, J. A. (2020). COVID-19, adulto mayor y edadismo: errores que nunca han de volver a ocurrir. Revista Española de Geriatría y Gerontología, 55(4), 191-192. https: / / doi.org/ 10.1016/ j.regg. 2020.04.001

Tejada Medina, V., Díaz Caro, C., González García, C., \& Ruiz Montero, P. (2020). Programas de intervención física en mujeres mayores a través del método Pilates: Una revisión sistemática. Retos, 39, 1006-1016. https: / / doi.org/ 10.47197/ retos.v0i39.78005

Tornero-Q uiñones, I., Sáez-Padilla, J., EspinaDíaz, A ., A bad Robles, M. T., \& Sierra Robles, Á. (2020). Functional Ability, Frailty and Risk of Falls in the Elderly: Relations with Autonomy in Daily Living. International Journal of Environmental Research and Public H ealth, 17 (3), 1006. https:/ / doi.org/ 10.3390/ ijerph17031006

U rzúa, Alfonso, Vera-Villarroel, Pablo, Caqueo-U rízar, Alejandra, \& Polanco-Carrasco, Roberto. (2020). La Psicología en la prevención y manejo del COVID-19. A portes desdelaevidencia inicial. Terapia Psicológica, 38(1), 103-118. https:/ / dx. doi.org/ 10.4067/ S0718-48082020000100103

Wang, D., Wang, P., Lan, K., Zhang, Y., \& Pan, Y. (2020). Effectiveness ofTai chi exercise on overall quality of life and its physical and psychological componentsamong older adults: a systematic review and meta-analysis. Brazilian Journal of M edical and Biological Research, 53(10), e10196. https:/ / doi.org/ 10.1590/ 1414-431X202010196

Woods, J. A., Hutchinson, N. T., Powers, S. K., Roberts, W. O., Gomez-Cabrera, M. C., Radak, Z., Berkes, I., Boros, A., Boldogh, I., Leeuwenburgh, C., Coelho-Júnior, H. J., M arzetti, E., Cheng,Y., Liu, J., Durstine, J. L., Sun, J., \& Ji, L. L. (2020). The COVID-19 pandemic and physical activity. SportsM edicine and Health Science, 2(2), 55-64. https:/ / doi.org/ 10.1016/ j.smhs.2020.05.006

Yamada, M., Kimura, Y., Ishiyama, D., O tobe, Y., Suzuki, M., Koyama, S. , Kikuchi, T., Kusumi, H. , \&A rai, H. (2020). Effect of the COVID-19 Epidemic on Physical Activity in CommunityDwelling Older Adults in Japan: A Cross-Sectional O nline Survey. The Journal of Nutrition, Health \& Aging, 1-3. Advance online publication. https:/ / doi.org/ 10.1007/ s12603-020$1424-2$

Ye, P., Liu, Y., Zhang, J., Peng, K., Pan, X., Shen, Y., Xiao, S., Armstrong, E. , Er,Y., Duan, L., Ivers, R., Keay, L., \&Tian, M. (2020). Falls prevention interventionsfor community-dwelling older people living in mainland China: a narrative systematic review. BMC H ealth Services Research, 20(1), 808. https:/ / doi. org/ 10.1186/ s12913-020-05645-0 